05.2

\title{
Эффективное возбуждение магнитоэлектрического эффекта В структуре ферромагнетик-пьезоэлектрик током через магнитный слой
}

\author{
(ㄱ Д.А. Бурдин, Д.В. Савельев, Н.А. Экономов, Д.В. Чашин, Ю.К. Фетисов \\ МИРЭА - Российский технологический университет, Москва, Россия \\ E-mail: phantastic@mail.ru
}

Поступило в Редакцию 24 декабря 2019г.

В окончательной редакции 24 декабря 2019 г.

Принято к публикации 28 февраля 2020 г.

Продемонстрировано эффективное возбуждение магнитоэлектрического эффекта в планарной структуре аморфный ферромагнетик-пьезоэлектрический цирконат-титанат свинца переменным током, протекающим через магнитный слой. Из-за отсутствия размагничивания и ориентации возбуждающего магнитного поля перпендикулярно постоянному полю структура генерирует четные гармоники напряжения с эффективностью, в несколько раз превышающей эффективность возбуждения эффекта объемными катушками. Показано, что амплитуда второй гармоники пропорциональна пьезомагнитному коэффициенту магнитного слоя, квадратично растет с амплитудой тока и убывает обратно пропорционально магнитному полю смещения.

Ключевые слова: магнитоэлектрический эффект, магнитострикция, пьезоэффект, генерация гармоник.

DOI: 10.21883/PJTF.2020.10.49428.18165

Магнитоэлектрический (МЭ) эффект в планарных композитных структурах с ферромагнитными и пьезоэлектрическими слоями возникает из-за взаимной связи намагниченности ферромагнетика с поляризацией пьезоэлектрика через их совместную механическую деформацию [1]. Прямой МЭ-эффект проявляется в генерации структурой переменного напряжения $u$ при помещении ее в возбуждающее переменное магнитное поле $h$ и параллельное ему постоянное магнитное поле смещения $H$. Возбуждающее магнитное поле на практике создают с помощью электромагнитных катушек, охватывающих структуру [2], что существенно увеличивает размеры устройств, использующих МЭ-эффект, и ограничивает сверху (из-за индуктивности катушек) диапазон рабочих частот таких устройств. Поэтому актуальной задачей является разработка альтернативных и более эффективных методов возбуждения МЭ-эффекта в композитных структурах.

В [3] наблюдался МЭ-эффект в структуре со слоями из аморфного ферромагнетика (FM) и пьезоэлектрического цирконата-титаната свинца (PZT) при пропускании переменного тока $I(f)$ через полосок, расположенный вблизи ее поверхности, и намагничивании полем $H$ вдоль оси структуры. Показано, что при совпадении частоты тока $f$ с частотой изгибных или планарных колебаний структуры она генерирует напряжение той же частоты с максимальной эффективностью до $u / I \approx 0.25 \mathrm{~V} / \mathrm{A}$. В [4,5] МЭ-эффект обнаружен в структуре FM-PZT, намагниченной вдоль оси полем $H$, при пропускании тока непосредственно через магнитный слой структуры. Наблюдалась генерация напряжения на частоте акустического резонанса структуры при возбуждении током с половинной частотой. Эффективность генерации второй гармоники напряжения составляла $u / I \sim 0.05 \mathrm{~V} / \mathrm{A}$ и росла с увеличением поля $H$. Однако в этих работах не рассмотрены особенности возбуждения нелинейного МЭ-эффекта током и не приведено теоретическое описание эффекта.

В настоящей работе экспериментально продемонстрировано эффективное возбуждение гармоник МЭ-напряжения в двухслойной структуре FM-PZT переменным током, протекающим непосредственно через магнитный слой структуры, и приведена теория, качественно объясняющая полевые и амплитудные зависимости амплитуд гармоник.

В измерениях была использована структура, схематически изображенная на рис. 1. Она содержала слой из аморфного ферромагнетика состава FeBSiC (Metglas $2605 \mathrm{~S}$ ) длиной $L=33 \mathrm{~mm}$, шириной $b_{m}=1.5 \mathrm{~mm}$ и толщиной $a_{m}=20 \mu \mathrm{m}$ и слой из PZT-керамики с Ag-электродами состава $\mathrm{Pb}\left(\mathrm{Zr}_{0.52} \mathrm{Ti}_{0.48}\right) \mathrm{O}_{3}$ длиной $33 \mathrm{~mm}$, шириной $1.5 \mathrm{~mm}$ и толщиной $a_{p}=300 \mu \mathrm{m}$. Магнитострикция насыщения FM-слоя достигала $\lambda_{S} \approx 23 \cdot 10^{-6}$ в поле насыщения $H_{S} \approx 8 \mathrm{kA} / \mathrm{m}$, а пьезомодуль керамики

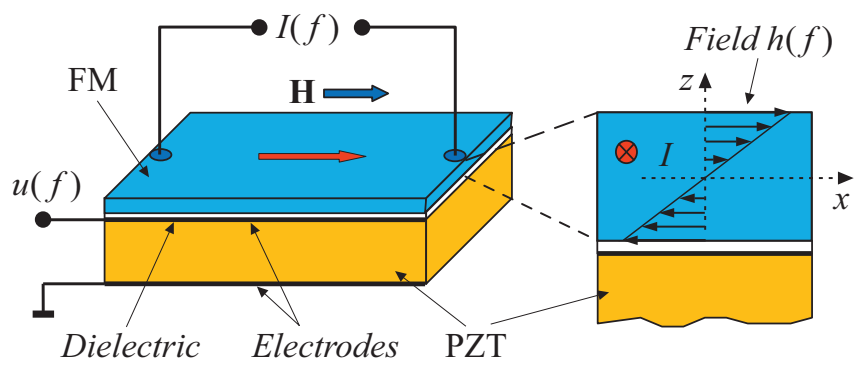

Pис. 1. Схематическое изображение структуры ферромагнетик-пьезоэлектрик (FM-PZT). 


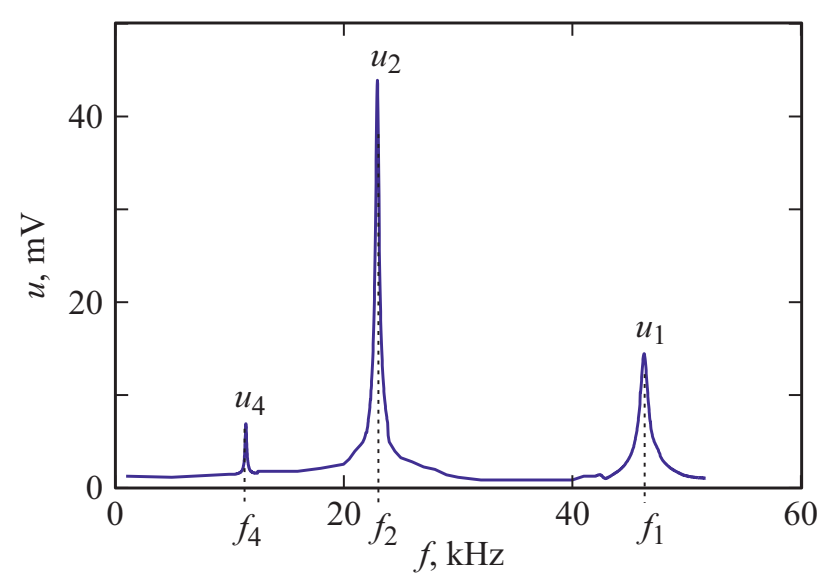

Рис. 2. Зависимость МЭ-напряжения $и$, генерируемого структурой $\mathrm{FM}-\mathrm{PZT}$, от частоты $f$ тока через магнитный слой при поле смещения $H=50 \mathrm{~A} / \mathrm{m}$ и $I=200 \mathrm{~mA}$.

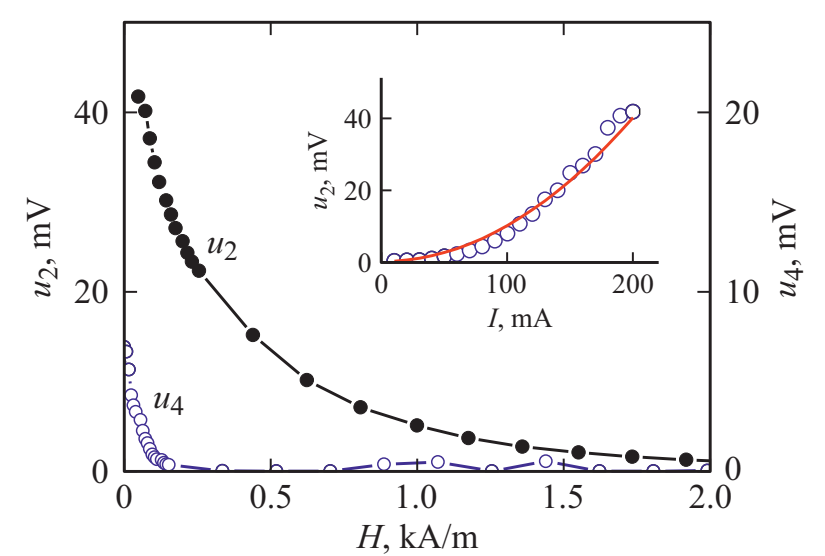

Рис. 3. Зависимость амплитуд второй $u_{2}$ и четвертой $u_{4}$ гармоник МЭ-напряжения от поля $H$ при $I=200 \mathrm{~mA}$. На вставке показана зависимость $u_{2}$ от тока $I$ при $H=50 \mathrm{~A} / \mathrm{m}$, сплошная линия - квадратичная аппроксимация.

равнялся $d_{31} \approx 175 \mathrm{pC} / \mathrm{N}$. На электроды PZT-слоя был нанесен слой диэлектрика толщиной $\sim 2 \mu \mathrm{m}$, обеспечивающий электрическую развязку слоев. Затем слои структуры были соединены под прессом с помощью цианакрилатного клея. Структура была закреплена в центре на жестком стержне и помещена между полюсами электромагнита в постоянное магнитное поле $H=0-40 \mathrm{kA} / \mathrm{m}$, направленное вдоль ее длинной стороны. Через FM-слой пропускался переменный ток с амплитудой до $I=200 \mathrm{~mA}$ и частотой $f=0-100 \mathrm{kHz}$ от генератора Agilent 33210a. Напряжение $u$, генерируемое в результате МЭ-эффекта между электродами РZТ-слоя, регистрировалось с помощью синхронного усилителя SR850 и мультиметра FLUKE 45.

На рис. 2 показана зависимость амплитуды генерируемого структурой напряжения $u$ от частоты возбуждающего тока $f$ при $I=200 \mathrm{~mA}$ и $H=50 \mathrm{~A} / \mathrm{m}$. Видны три пика с частотами $f_{1} \approx 46.3 \mathrm{kHz}, f_{2}=f_{1} / 2 \approx 23.0 \mathrm{kHz}$, $f_{4}=f_{1} / 4 \approx 11.5 \mathrm{kHz}$ и примерно одинаковыми доб- ротностями $Q \approx 80$. Проверено, что генерируемое структурой напряжение $u$ на всех трех пиках имело одну и ту же частоту $f_{1} \approx 46.3 \mathrm{kHz}$. Оценка по формуле $f=1 /(2 L) \sqrt{Y / \rho}$ (где эффективные модуль Юнга $Y=\left(Y_{m} a_{m}+Y_{p} a_{p}\right) /\left(a_{m}+a_{p}\right)$ и плотность $\left.\rho=\left(\rho_{m} a_{m}+\rho_{p} a_{p}\right) /\left(a_{m}+a_{p}\right)\right)$ при значениях параметров слоев $Y_{p}=7 \cdot 10^{10} \mathrm{~N} / \mathrm{m}^{2}, Y_{m}=7 \cdot 10^{10} \mathrm{~N} / \mathrm{m}^{2}$, $\rho_{p}=7.7 \cdot 10^{3} \mathrm{~kg} / \mathrm{m}^{3}, \quad \rho_{m}=8.2 \cdot 10^{3} \mathrm{~kg} / \mathrm{m}^{3}$ дала частоту продольного акустического резонанса структуры $f_{\text {cal }} \approx 47.8 \mathrm{kHz}$, хорошо совпавшую с $f_{1}$. Отсюда следует, что пик при частоте возбуждающего тока $f_{1}$ отвечает резонансной генерации основной гармоники МЭ-напряжения с амплитудой $u_{1}$, пик на частоте тока $f_{2}-$ генерации второй гармоники с амплитудой $u_{2}$, а пик на частоте тока $f_{4}-$ генерации четвертой гармоники с амплитудой $u_{4}$ (подробнее см. в [6]). Отметим, что структура наиболее эффективно генерирует вторую гармонику напряжения, что наблюдали и авторы работы [4].

На рис. 3 приведены зависимости амплитуд второй $u_{2}$ и четвертой $u_{4}$ гармоник МЭ-напряжения от постоянного магнитного поля $H$ при амплитуде тока $I=200 \mathrm{~mA}$. Вторую гармонику возбуждали током с частотой $23 \mathrm{kHz}$, а четвертую - током с частотой $11.5 \mathrm{kHz}$. Видно, что вторая гармоника имеет максимальную амплитуду $u_{2} \approx 42.3 \mathrm{mV}$ в отсутствие поля и затем монотонно спадает приблизительно по закону $u_{2} \sim 1 / H$. Четвертая гармоника имеет максимальную амплитуду $u_{4} \approx 7 \mathrm{mV}$ при $H=0$, а затем спадает до нуля значительно быстрее, чем вторая гармоника. На вставке к рис. 3 также показано, что амплитуда второй гармоники квадратично растет с увеличением возбуждающего тока: $u_{2} \sim I^{2}$.

Для объяснения особенностей МЭ-эффекта в структуpe с током через FM-слой найдем поле $h$, создаваемое током в слое. Считаем, что плотность тока $j=I /\left(a_{m} b_{m}\right)$ равномерна по сечению $x-z$ FM-слоя. Используя закон полного тока, получаем $h(z)=I z /\left(a_{m} b_{m}\right)$, т.е. поле $h$ равно нулю на средней линии слоя при $z=0$, линейно изменяется по толщине слоя, направления поля противоположны на его поверхностях при $z= \pm a_{m} / 2$ (рис. 1). При $I=200 \mathrm{~mA}$ амплитуда поля максимальна на поверхностях слоя $\left(h_{\max } \approx 66 \mathrm{~A} / \mathrm{m}\right)$, а средняя по толщине слоя амплитуда поля равна $33 \mathrm{~A} / \mathrm{m}$. Это магнитное поле приводит к магнитострикционной деформации FM-слоя вдоль оси $x$. Поскольку деформация зависит квадратично от поля, она имеет одинаковый знак по всей толщине FM-слоя и через границу раздела передается PZT-слою структуры.

В отличие от случая возбуждения МЭ-эффекта внешним магнитным полем при возбуждении МЭ-эффекта током, протекающим непосредственно через FM-слой, эффекты размагничивания отсутствуют и не ослабляют поле внутри ферромагнетика. Это в свою очередь приводит к более высокой эффективности возбуждения МЭ-эффекта током. В нашем случае эффективность генерации второй гармоники составляла $u / I \approx 0.22 \mathrm{~V} / \mathrm{A}$, т.е. в $\sim 4$ раза больше, чем в [4]. Используя данные 
рис. 2 и среднее поле $h=33 \mathrm{~A} / \mathrm{m}$ при $I=200 \mathrm{~mA}$, находим нелинейный МЭ-коэффициент для второй гармоники $\alpha_{E}^{(2)}=u_{2} /\left(h^{2} a_{p}\right) \approx 0.13 \Omega \cdot \mathrm{m}$, который в $\sim 1.5$ раза выше, чем при возбуждении второй гармоники катушкой [7].

Из рис. 3 видно, что форма полевой зависимости амплитуды второй гармоники $u_{2}(H)$ при возбуждении МЭ-эффекта током через FM-слой качественно отличается от формы аналогичной зависимости в случае возбуждения МЭ-эффекта полем катушки [6,7]. Для объяснения воспользуемся результатами работы [7], где показано, что МЭ-напряжение дается приближенной формулой $u(H)=A d_{31} \lambda(H)$. Здесь $A-$ коэффициент, определяемый формой, размерами и диэлектрическими параметрами слоев структуры, $d_{31}$ - пьезомодуль, $\lambda(H)-$ зависимость магнитострикции от поля $H$. Переменный ток $I \cos (2 \pi f t)$ создает в FM-слое поле $h \cos (2 \pi f t)$, направленное перпендикулярно полю $H$. Тогда изменение поля внутри ферромагнетика при слабом возбуждении $(h \ll H)$ равно $\delta H=\sqrt{H^{2}+h^{2}}-H \approx h^{2} / H$. Раскладывая $u(H)$ в ряд Тейлора вблизи $H$ до членов второго порядка малости и оставляя в результате только гармонические слагаемые, после преобразований получаем

$$
\begin{aligned}
& u(f) \approx A d_{31}\left(\lambda^{(1)} \frac{h^{2}}{2 H}+\lambda^{(2)} \frac{h^{4}}{4 H^{2}}\right) \cos (4 \pi f t) \\
& +A d_{31} \lambda^{(2)} \frac{h^{4}}{16 H^{2}} \cos (8 \pi f t)+\ldots
\end{aligned}
$$

где $\lambda^{(1)}(H)=\partial \lambda / \partial H-$ пьезомагнитный коэффициент, $\lambda^{(2)}(H)=\partial^{2} \lambda / \partial H^{2}-$ нелинейный пьезомагнитный коэффициент.

Из (1) следует, что при возбуждении током структура должна генерировать только четные гармоники напряжения; амплитуда второй гармоники содержит два слагаемых, причем первое $u_{2} \approx A d_{31} \lambda^{(1)} h^{2} /(2 H)$ является определяющим, поскольку $\lambda^{(1)}$ на порядок больше $\lambda^{(2)}$ [7]; амплитуда четвертой гармоники $u_{4} \sim \lambda^{(2)} h^{4} / H^{2}$ быстро падает с увеличением $H$. Предсказываемая теорией высокая эффективность генерации второй гармоники МЭ-напряжения, ее зависимость от постоянного поля вида $u_{2} \sim 1 / H$ и квадратичная зависимость от возбуждающего поля $u_{2} \sim h^{2}$ хорошо согласуются с данными измерений, приведенными на рис. 2 и 3. Причиной генерации первой гармоники МЭ-напряжения, обнаруженной экспериментально (рис. 2), может быть неортогональность полей $h$ и $H$ из-за магнитной анизотропии FM-слоя структуры.

Таким образом, показано, что переменный ток, протекающий через магнитный слой структуры ферромагнетик-пьезоэлектрик, из-за отсутствия влияния размагничивания эффективно возбуждает четные гармоники МЭ-напряжения. Зависимость амплитуды второй гармоники напряжения от постоянного магнитного поля при этом качественно отличается от аналогичной зависимости при возбуждении МЭ-эффекта внешним магнитным полем катушки. Использование описанного метода возбуждения позволит существенно уменьшить размеры и расширить диапазон рабочих частот МЭ-устройств.

\section{Финансирование работы}

Работа поддержана Министерством науки и высшего образования РФ (грант МК-2639.2018.2) и Российским фондом фундаментальных исследований (грант 19-07-00594/19).

\section{Конфликт интересов}

Авторы заявляют, что у них нет конфликта интересов.

\section{Список литературы}

[1] Srinivasan G. // Annu. Rev. Mater. Res. 2010. V. 40. P. 153178. DOI: 10.1146/annurev-matsci-070909-104459.

[2] Wu Z., Bian L., Wang S., Zhang $X$. // Sensors Actuators A. 2017. V. 262. P. 108-113. DOI: 10.1016/j.sna.2017.04.049

[3] Chashin D.V., Fetisov L.Y., Saveliev D.V., Fetisov Y.K. // IEEE Sensors Lett. 2019. V. 3. P. 2500804. DOI: 10.1109/LSENS.2019.2895966

[4] Prieto J.L., Aroca C., Lopez E., Sanchez M.C., Sanchez P. // J. Magn. Magn. Mater. 2000. V. 215-216. P. 756-758. DOI: 10.1016/S0304-8853(00)00279-1

[5] Burdin D.A., Saveliev D.V., Fetisov Y.K., Ekonomov N.A., Chashin D.V. // J. Phys.: Conf. Ser. 2019. V. 1389. P. 012049. DOI: 10.1088/1742-6596/1389/1/012049

[6] Fetisov L.Y., Burdin D.A., Ekonomov N.A., Chashin D.V. Zhang J., Srinivasan G., Fetisov Y.K. // J. Phys. D: Appl. Phys. 2018. V. 51. P. 154003. DOI: $10.1088 / 1361-6463 /$ aab384

[7] Burdin D.A., Chashin D.V., Ekonomov N.A., Fetisov L.Y., Fetisov Y.K., Sreenivasulu G., Srinivasan G. // J. Magn. Magn. Mater. 2014. V. 358-359. P. 98-104. DOI: $10.1016 /$ j.jmmm.2014.01.062 